\title{
A large-area, all-plastic, flexible electroosmotic pump
}

\author{
Katarina Bengtsson ${ }^{1,2} \cdot$ Nathaniel D. Robinson ${ }^{1,2}$ (])
}

Received: 26 July 2017 / Accepted: 6 November 2017 / Published online: 13 November 2017

(c) The Author(s) 2017. This article is an open access publication

\begin{abstract}
A large-area, fabric-like pump would potentially have applications, for example, in controlling water transport through a garment, such as a rain jacket, regardless of the external temperature and humidity. This paper presents an all-plastic, flexible electroosmotic pump, constructed from commercially available materials: A polycarbonate membrane combined with the electrochemically active polymer poly(3,4-ethylenedioxythiophene) polystyrene sulfonate that actively transports water using an electric potential that can be supplied by a small battery. By using electrochemically active polymer electrodes instead of metal electrodes, the electrochemical reaction that drives flow avoids the oxygen and hydrogen gas production or $\mathrm{pH}$ changes associated with water electrolysis. We observe a water mass flux up to $23 \mathrm{mg} \mathrm{min}^{-1}$ per $\mathrm{cm}^{2}$ polycarbonate membrane (porosity 10-15\%), at an applied potential of $5 \mathrm{~V}$, and a limiting operating pressure of $0.3 \mathrm{kPa} \mathrm{V}^{-1}$, similar to previously reported membrane-based electroosmotic pumps.
\end{abstract}

Keywords Electroosmosis $\cdot$ Conducting polymer $\cdot$ Conjugated polymer $\cdot$ PEDOT:PSS $\cdot$ Track-etched porosity

\section{Introduction}

There are many applications where flexible fabric-like pumps capable of transporting fluid from one side of a textile to the other would be useful. This technology could, for example, be applied in specialized clothing, capable of transporting water from inside the garment to the outside, even when the air outside the garment is saturated with water vapor. Similar opportunities exist in medicine, where the current technology (e.g., a plaster cast for immobilizing a broken bone) traps moisture (sweat) against the skin, leading to irritation and potentially infection. Electroosmotic pumps (EOPs), unlike most other types of pumps, are well-suited to these applications because they operate without moving parts and can be designed and fabricated to operate over relatively large areas. In this work, we present a plastic EOP

Electronic supplementary material The online version of this article (https://doi.org/10.1007/s10404-017-2017-1) contains supplementary material, which is available to authorized users.

Nathaniel D. Robinson

natro@ifm.liu.se

1 Transport and Separations Group, Department of Physics, Chemistry and Biology, Linköping University, Linköping, Sweden

2 LunaMicro AB, Linköping, Sweden incorporating large-capacity conducting polymer electrodes and a flexible porous membrane, resulting in a highly flexible fabric-like device that operates with almost no water electrolysis. We believe these pumps have the potential for future use in areas such as clothing and medical devices.

Today, water removal, in the case of functional clothing, is mainly achieved through passive vapor transport through halogenated semi-permeable membranes. This process depends strongly on the external environmental conditions, primarily the relative humidity, but also the temperature. One published exception offers a fabric-like pump which electroosmotically controls water transport (Eidsnes et al. 2004; Heldal and Lauper 2013). EOPs employ electroosmosis, where a liquid's movement, e.g., in a capillary, is driven by an externally applied electric field (Kirby 2010). The motion of the liquid stems from the coulombic forces on the ions in the so-called double layer-a thin layer of ions that forms near a charged wall in contact with an electrolyte. The net charge in this ionic layer depends on the electrolyte and the chemistry of the surface, and directly affects the velocity of flow generated per unit of electric field (the electroosmotic mobility). These pumps are most commonly associated with analytic chemistry and microfluidics, since they are compact, easily integrated into small devices, and can be implemented in a wide range of applications for micro-high-pressure liquid chromatography ( $\mathrm{HPLC}$ ), 
capillary electrophoresis, chip-based assays, drug delivery, and device actuation (Chen et al. 2004; Glawdel and Ren 2009; Li and Harrison 1997; Litster et al. 2010; Manz et al. 1994; Wang et al. 2009; Zeng et al. 2001).

As stated above, the fluid flow through an EOP is driven by an externally applied field. Since the fluid being pumped functions (to a degree depending on its composition) as an electrolyte, electrochemical (Faradaic) reactions must occur at the electrolyte-electrode interfaces in order to maintain an electric field within the electrolyte. Without these reactions, the ions attracted to each impenetrable electrode interface accumulate, concentrating the electric field locally until the bulk of the electrolyte (and active part of the pump) is effectively shielded from the field (Bard and Faulkner 2001). The most common electrochemical reaction in an aqueous system with metal electrodes is water electrolysis, which produces gas and causes $\mathrm{pH}$ changes as illustrated in half-reactions (1) and (2). Both the $\mathrm{pH}$ changes (generated $\mathrm{H}^{+}$and $\mathrm{OH}^{-}$) and the gas evolution are detrimental to the pump's operation. Changes in $\mathrm{pH}$ influence the chemistry (e.g., protonation) of the surface of the narrow channels within the pump, changing the surface charge density (alternatively the zeta potential), and consequently the electroosmotic mobility. Variations in $\mathrm{pH}$ can also influence any chemical or biological species in the sample being pumped. Oxygen and hydrogen gas generated at the electrodes can create bubbles that hinder the electrolyte's contact with the electrode itself. In the worst case, the accumulation of gas at the electrode-electrolyte interface breaks the electrochemical circuit, rendering the pump inoperable. Many EOPs therefore require specialized mechanisms for handling the by-products of water electrolysis, e.g., using a pH buffer, gas collection systems or driving EOPs at very low voltages (Brask et al. 2005; Heuck and Staufer 2011; Lin et al. 2007; Yao et al. 2003).

Oxidation

$2 \mathrm{H}_{2} \mathrm{O} \rightarrow \mathrm{O}_{2(\mathrm{~g})}+4 \mathrm{H}_{(\mathrm{aq})}^{+}+4 \mathrm{e}^{-}$

Reduction

$2 \mathrm{H}_{2} \mathrm{O}+2 \mathrm{e}^{-} \rightarrow \mathrm{H}_{2(\mathrm{~g})}+2 \mathrm{OH}_{(\mathrm{aq})}^{-}$

Instead of driving solvent electrolysis, the Faradaic reactions at the metal electrodes can be accomplished within the electrode material itself if this material can be suitably oxidized and reduced. The conducting polymer poly $(3,4-$ ethylenedioxythiophene), often blended with polystyrene sulfonate (PEDOT:PSS), is an example of an electrode material that can undergo electrochemistry without releasing detrimental by-products (Bengtsson et al. 2014; Erlandsson and Robinson 2011). The reversible oxidation of PEDOT:PSS can be seen in reaction scheme (3), where $\mathrm{M}^{+}$represents nearly any cation, e.g., $\mathrm{Na}^{+}$, which moves between the electrolyte and electrode bulk (which is typically a hydrogel). The availability of this reaction (and the corresponding reduction) dramatically reduces (or eliminates) water electrolysis. However, electrochemically active electrodes have a finite electrochemical capacity. This capacity defines the limits of device operation for which water electrolysis is minimized. Electrochemically active polymer electrodes are more flexible and easier to process than most metal electrodes. They can also be mass-produced without metals or rare earth elements, resulting in less expensive and more sustainable products than would be achievable with, for example, gold or platinum.

$\mathrm{PEDOT}^{0}+\mathrm{M}^{+} \mathrm{PSS}^{-} \rightleftarrows \mathrm{PEDOT}^{+} \mathrm{PSS}^{-}+\mathrm{M}^{+}$

There are previous examples of devices in which conducting polymers have been used instead of metal electrodes in EOPs. The "redox-conducting" polymer poly(hydroquinone/ benzoquinone) (PHBQ) was used in a membrane-based pump to reduce the effects of water electrolysis (Sachan et al. 2014). However, the authors reported that water electrolysis still became the dominating Faradaic reaction at increased applied potentials due to the slow reaction rate of oxidation or reduction of PHBQ. Similarly, polyanilinewrapped aminated graphene electrodes were used in combination with a silica frit to produce a long-lasting, non-gassing EOP (Kumar et al. 2015). Finally, we have previously shown poly(3,4-ethylenedioxythiophene) polystyrene sulfonate (PEDOT:PSS) as an electrode material to mitigate water electrolysis at applied potential as high as $250 \mathrm{~V}$ across (rigid) electrokinetic systems (Bengtsson et al. 2014; Erlandsson and Robinson 2011).

Aside from the electrodes, an EOP needs a capillary or porous media through which ions near the media's surface, driven by the electric field, drag solvent (Kirby 2010). Traditionally, most EOPs have been constructed with beds of packed silica particles (Zeng et al. 2001) or relatively rigid porous materials such as anodic aluminum oxide (Ai et al. 2010; Miao et al. 2007). Polymeric, porous membranes function well as this media in flexible low-voltage EOPs since they are relatively thin, mechanically flexible, and can be highly porous. The sub-mm thickness of these membranes results in a strong electric field (range $100-250 \mathrm{kV} \mathrm{m}^{-1}$ ), even when small potentials $(2-5 \mathrm{~V})$ are applied, resulting in significant flow even at potentials that are small enough to be supplied by, for example, standard AA batteries and used safely in consumer products. Several groups have previously shown that membranes of polycarbonate (PC), polyethylene terephthalate (PET), and nylon can be incorporated in EOPs (Kwon et al. 2012; Wang et al. 2012; Wu et al. 2016). PC membranes reportedly generate volumetric flow rates up to $120 \mu \mathrm{min}^{-1}$ per $\mathrm{cm}^{2}$ of membrane area and V of applied potential (Eidsnes et al. 2004; Kwon et al. 2012; Wang et al. 
2012). This concept has even been developed into a "fabric" targeted for applications in functional clothing (Heldal and Lauper 2013). However, the "fabric" (device) uses metal (gold) or carbon electrodes that do not themselves undergo electrochemistry, resulting in little control over gas generation and $\mathrm{pH}$ changes.

We demonstrate an all-plastic, flexible and low-voltage EOP consisting of electrochemically active polymer (PEDOT:PSS) electrodes and a porous polymeric membrane (track-etched polycarbonate), resulting in a "fabric" through which water is actively transported using potentials below $5 \mathrm{~V}$. Our choice of electrode material results in a non-gassing and $\mathrm{pH}$-stable EOP that is soft and formable, and can be applied in areas where more traditional pumps fail.

\section{Materials and methods}

All chemicals, unless stated otherwise, were analytical grade and obtained from Sigma-Aldrich. The pump is a 3-layer structure in which a thin, flexible polycarbonate membrane (white) is placed between two plastic electrodes (see Fig. 1). The electrodes were prepared by applying $0.1-0.2 \mathrm{~g} \mathrm{~cm}^{-1}$ (wet) PEDOT:PSS (Clevios S V3, Heraeus) on paper towels (TX609, TechniCloth ${ }^{\circledR}$, TW Texwipe ${ }^{\circledR}$ ). To evaporate the solvent and bond the PEDOT to the tissue, the electrodes were baked (Heraeus, Function Line oven) as follows; $50{ }^{\circ} \mathrm{C}$ for $2 \mathrm{~h}$, followed by ramping of $3.6{ }^{\circ} \mathrm{C} \mathrm{h}^{-1}-90{ }^{\circ} \mathrm{C}$, and last $90{ }^{\circ} \mathrm{C}$ for $5 \mathrm{~h}$, before letting them cool down to room temperature. The dried electrodes were thoroughly rinsed to remove, e.g., excess polystyrene sulfonate (PSS) or diethylene glycol by first submersing them in $0.1 \mathrm{M} \mathrm{NaCl}$ for $16 \mathrm{~h}$, and then, using them as a pair in a simple aqueous electrochemical cell with $0.1 \mathrm{M} \mathrm{NaCl}$ electrolyte driven at $\pm 0.6 \mathrm{~V}$ for $2 \mathrm{~min}$ at each polarity, repeated 3 times. The $\mathrm{pH}$ in the electrolyte was adjusted to $7 \pm 0.2$ between each step using $0.1 \mathrm{M} \mathrm{NaOH}$.

A track-etched polycarbonate membrane, coated with polyvinylpyrrolidone (PVP) $\left(\right.$ Cyclopore $^{\mathrm{TM}}$, Whatman ${ }^{\circledR}$, $0.4 \mu \mathrm{m}$ pore size, diameter $=47 \mathrm{~mm}$, thickness $=20 \mu \mathrm{m}$, porosity $9 \times 10^{7}$ pores $\mathrm{cm}^{-2}$ ), was mounted on Parafilm " $\mathrm{M}$ "® (Laboratory film, Bemis ${ }^{\circledR}$, Bemis flexible packaging) by lamination at $90^{\circ} \mathrm{C}$ on a hotplate (IKA ${ }^{\mathrm{R}} \mathrm{RH}$ digital). The active pumping area (free from parafilm) was $3.8 \pm 0.2 \mathrm{~cm}^{2}$, and the estimated porosity was between 10 and $15 \%$. The membrane was rinsed with $95 \%$ ethanol and deionized water prior to use in a pump.

Scanning electron microscopy (SEM) was performed on the track-etched membrane to verify the pore size and porosity. SEM was done using a LEO 1550 Gemini scanning electron microscope with an acceleration voltage of $5 \mathrm{kV}$. Prior to measurement, the samples were sputtered with 15 Å Pt using a Leica EM SCD500. (a)
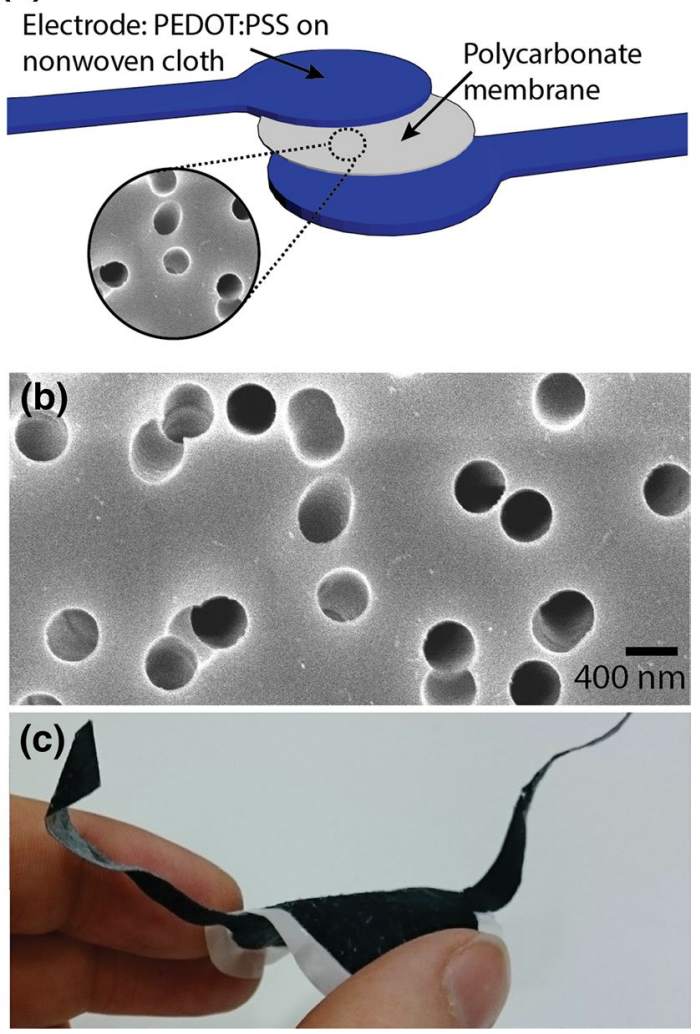

Fig. 1 Electroosmotic pump and inner membrane. a Schematic of the electroosmotic pump consisting of two electrodes (dark) sandwiching a porous membrane (white) including a magnification (SEM image) of the polycarbonate membrane, illustrating its porosity. b SEM of the track-etched polycarbonate membrane with scale bar. c A pump made from a Cyclopore ${ }^{\mathrm{TM}}$ Whatman $^{\circledR}$ (polycarbonate) membrane and two PEDOT:PSS electrodes (diameter $=32 \pm 0.2 \mathrm{~mm}$ ). The total thickness of the stack is $1.5 \pm 0.2 \mathrm{~mm}$

A schematic, cross-sectional image of the flow cell used for characterizing the flow through the pump can be seen in Fig. 2. The flow cell chamber, determined by the size of the O-rings, had a diameter of $34 \mathrm{~mm}$ and depth of $2 \mathrm{~mm}$. The body of the flow cell was 3-D printed out of acrylonitrile butadiene styrene (ABS) at Wematter AB, Linköping, Sweden. The pieces were treated with acetone to ensure that the surfaces were watertight. The electrodes were cut to fit within the flow cell (see Fig. 1), with a strip of the conducting material exiting the cell between the O-rings for electronic contact (see Fig. 2). To prevent electrolyte from being transported out of the cell through the electrical contacts, they were "sealed" by wicking melted paraffin wax into the paper. The O-rings and edges around the stack were coated with a thin layer of vacuum grease (Dow Corning ${ }^{\circledR}$, highvacuum grease GMBH).

The coulombic capacity of the electrode pair was estimated by applying a constant potential of $0.6 \mathrm{~V}$ for $20 \mathrm{~min}$ (in each polarization) to the stack mounted in the flow cell 


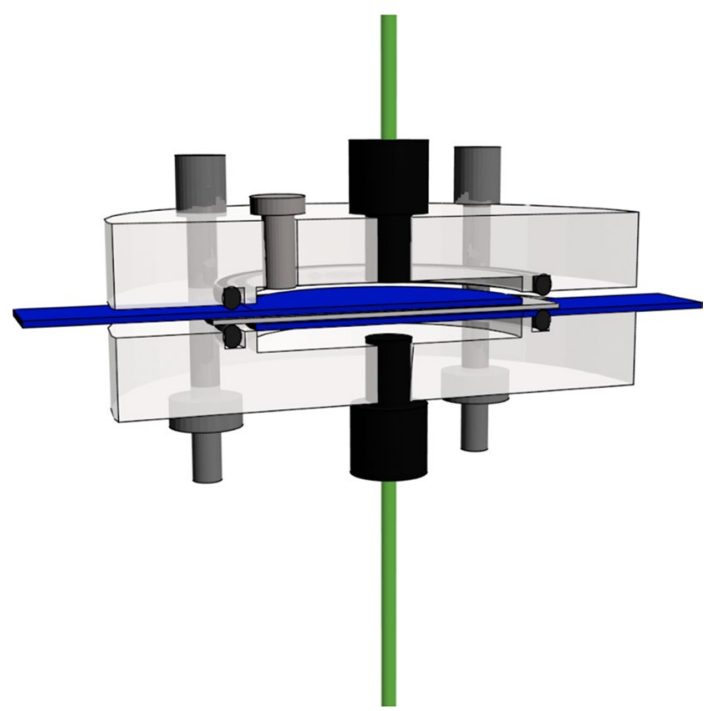

Fig. 2 Schematic cross-sectional image of the flow cell. The 3-D printed structure together with two O-rings (black) forms a tight seal around the stack forming a flow cell with a diameter defined by the O-rings. The flow cell is compressed with 6 bolts (gray, only 2 shown). The tubing is connected to the flow cell using M6 male "nuts" next to one of which an air vent screw (small gray plug, only one shown) is placed in each half. All components are approximately to scale, and the entire setup has a thickness of $2 \mathrm{~cm}$ and a diameter of $67 \mathrm{~mm}$

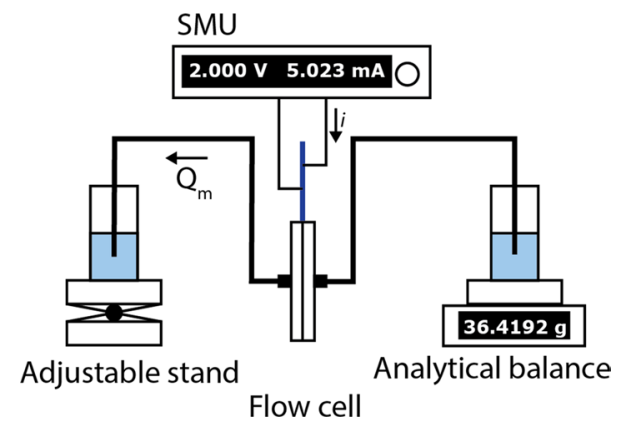

Fig. 3 Schematic of measurement setup. The source measurement unit (SMU, a Keithley 2636A) is connected to the pump stack mounted in the flow cell and either drives a constant current or applies a constant potential, inducing a mass flow $Q_{\mathrm{m}}$ through the flow cell. The pump in the flow cell transports fluid between two beakers through PTFE tubes, one of which is placed on a balance, the other on an adjustable stand

and then integrating the current measured. Each pair of PEDOT:PSS electrodes used in this study had a coulombic capacity in the range of $0.1-0.3 \mathrm{C}$.

A schematic of the overall measurement setup can be seen in Fig. 3. The fluid port on each side of the flow cell was connected to a parafilm-covered beaker using PTFE tubing (outer diameter $=1 / 16$ in., inner diameter $=0.75 \mathrm{~mm}$, length $=405 \mathrm{~mm}$, Supelco, Sigma-Aldrich). On the one side, the beaker was placed on an adjustable stand and on the other side, on a balance (VWR, La124i). The height of the stand was adjusted until the water level in the beaker matched that of the beaker on the balance as confirmed by the cessation of (pressure-driven) flow through the flow cell, i.e., until the mass in the beaker on the balance stopped changing. The mass of water in the beaker on the balance was recorded electronically with a computer via an RS232 interface. The mass flow rate through the cell was estimated from a linear regression of the recorded mass versus time followed by conversion to volumetric flow rate using a liquid density of $997 \mathrm{~g} \mathrm{~m}^{-3}$. A source measure unit (SMU) (Keithley 2636A, Tektronix Company) was used either to apply a constant potential $(0.3-5 \mathrm{~V})$ or to drive a constant current $(0.3-5 \mathrm{~mA})$ between the polymer electrodes. Measurements were performed at atmospheric pressure and room temperature. Before measuring, the flow cell was flushed with $10 \mathrm{ml}$ of $1 \mathrm{mM} \mathrm{NaOH}_{(\mathrm{aq})}$ followed by $100 \mathrm{ml}$ electrolyte, and the $\mathrm{pH}$ was measured before and after each set of experiments.

For a few experiments, the potential drop across the electrodes and membrane was measured using 2 sets of $\mathrm{Ag} / \mathrm{AgCl}$ reference electrodes, one placed in each beaker, and measuring the potential while maintaining zero current, using the second channel of the Keithley 2636A, while simultaneously applying a constant potential between the polymer electrodes with the first channel, where the only connection between reference electrodes and polymer electrodes was the electrolyte.

The limiting hydrostatic pressure that the pump is able to produce at each applied potential was determined by adjusting the difference in height between the beakers until there was no detectable mass change on the balance (no flow). The maximum potential for which the limiting pressure was obtained was $2 \mathrm{~V}$ due to the difficulty of finding the position of the stand at which zero net flow was achieved without exceeding the coulombic capacity of the electrodes when larger potentials were used. The limiting pressure was then calculated based on the difference in height between the water levels in the two beakers.

\section{Results and discussion}

This flexible, all-plastic pump can transport several hundreds of $\mu \mathrm{l}$ of water within minutes. Water and solutions with low salt concentration (1-10 mM) can be observed moving back and forth through a stack, such as the one shown in Fig. 1, outside the flow cell within a minute of applying a potential between 1 and $5 \mathrm{~V}$. Water is pumped through the stack even after the pump is bent, e.g., as shown in Fig. 1. A short video demonstrating fluid motion through the stack is available online (Supplementary Information 1). 
The mass flux (calculated per unit membrane area), as well as the volumetric flow rate $Q$, generated by a typical stack during operation at constant current can be seen in Fig. 4. The maximum mass flux was 25 and $5 \mathrm{mg} \mathrm{min}^{-1} \mathrm{~cm}^{-2}$ for $1 \mathrm{mM} \mathrm{NaCl}$ and $10 \mathrm{mM} \mathrm{NaCl}$, respectively, each at a current density $J$ of $1.3 \mathrm{~mA} \mathrm{~cm}{ }^{-2}$. The flow rate increases linearly with increasing current density and decreases with increasing salt concentration, as expected (Kirby 2010). This setup generated easily measurable flow with $10 \mathrm{mM} \mathrm{NaCl}$ electrolyte, but not for $100 \mathrm{mM} \mathrm{NaCl}$ electrolyte.

Figure 5 shows the mass flux (calculated per unit membrane area) and the volumetric flow rate versus applied potential as well as the average of the current density measured for each applied potential. The maximum mass flux was measured to be $28 \mathrm{mg} \mathrm{min}^{-1} \mathrm{~cm}^{-2}$, using $1 \mathrm{mM} \mathrm{NaCl}$ at $5 \mathrm{~V}$. Similarly arranged measurements using other brands of track-etched polycarbonate membranes are reported to have flow rates (normalized by total cross-sectional pore area) up to $120 \mu \mathrm{min}^{-1} \mathrm{~cm}^{-2}$ per volt of applied potential, pumping deionized water (Kwon et al. 2012; Wang et al. 2012). For our membrane, we estimate a corresponding maximum normalized volumetric flow rate of $50 \mu \mathrm{min}^{-1} \mathrm{~cm}^{-2}$ per $\mathrm{V}$ of applied potential for $1 \mathrm{mM} \mathrm{NaCl}, \mathrm{pH} 5.6$, similar to that reported for a polycarbonate membrane in $1 \mathrm{mM}$ borate buffer at pH 9 (Kwon et al. 2012).

Operating the pump using either constant potential or constant current can result in similar mass transport rates. However, the mass transport rate decreases while operating the pump using a constant potential for longer than $2 \mathrm{~min}$, compared to a constant rate when driving constant current. The nonlinear relationship apparent in Fig. 5 is likely caused by the increase in resistance of the PEDOT cathode [PEDOTs conductivity decreases as it is dedoped, see (Johansson et al. 2002; Nilsson et al. 2002)] and the "consumption" of the polymer available for oxidation/reduction during longer periods of operation (in the same direction) at elevated potential. Otherwise, the relationship between $J$ and $E$ depends very little on whether the pump is operated by
Fig. 4 a Mean mass flux based on total membrane area (porosity $12 \%$ ) and corresponding volumetric flow rate $Q$ (unfilled symbols) versus current density $J$. b Mean and standard error (bars) of applied potential $E$ during each pulse versus current density $J$ corresponding to the data shown in part a. Electrolyte was $1 \mathrm{mM} \mathrm{NaCl}$ (squares) and $10 \mathrm{mM} \mathrm{NaCl}$ (triangles) each with $\mathrm{pH}=5.7 \pm 0.2$ (a)

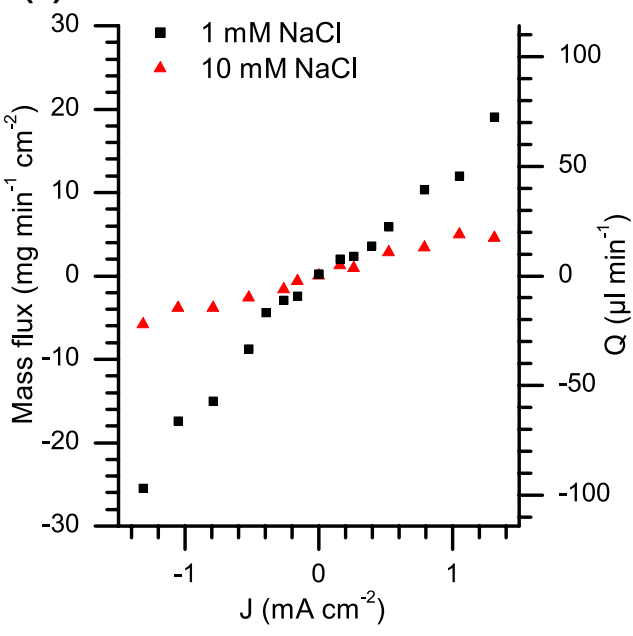

Fig. 5 a Mass flux based on membrane area (porosity $12 \%$, left-hand axis) and volumetric flow rate $Q$ (right-hand axis) versus applied potential $E$. Line represents best-fit curve with intercept fixed at the origin and slope $6.1 \mathrm{mg} \mathrm{min}^{-1} \mathrm{~cm}^{-2} \mathrm{~V}^{-1}$. b Mean current density $J$ versus applied potential. Both measurements were performed with $1 \mathrm{mM} \mathrm{NaCl}_{(\mathrm{aq})}, \mathrm{pH}=5.7 \pm 0.2$ (a)

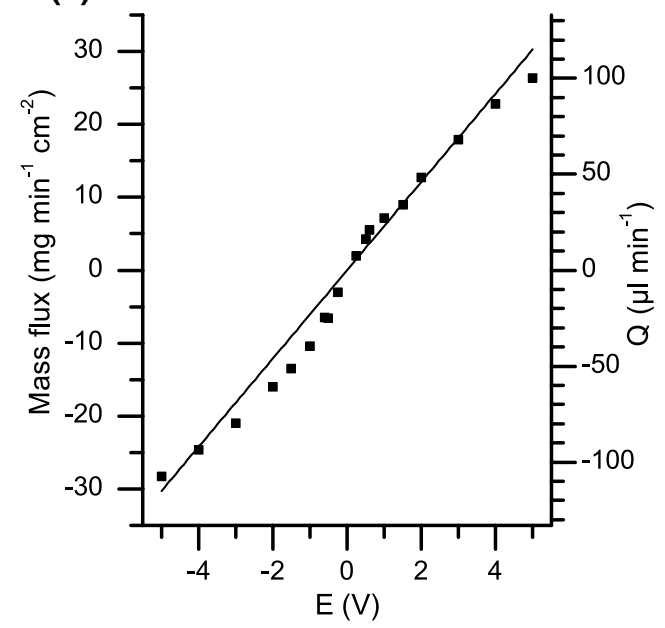

(b)

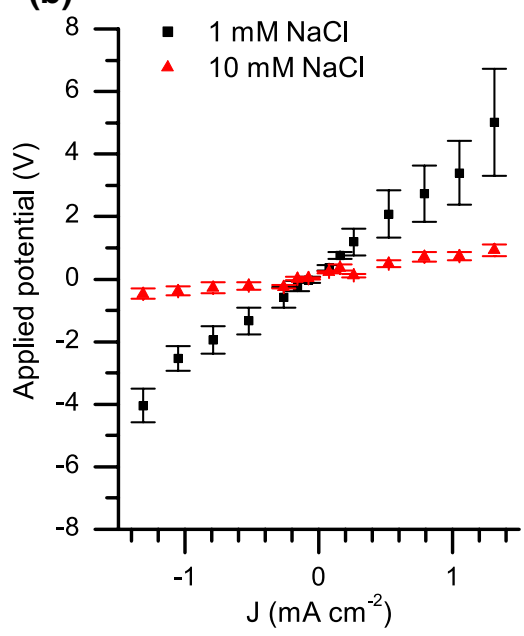

(b)

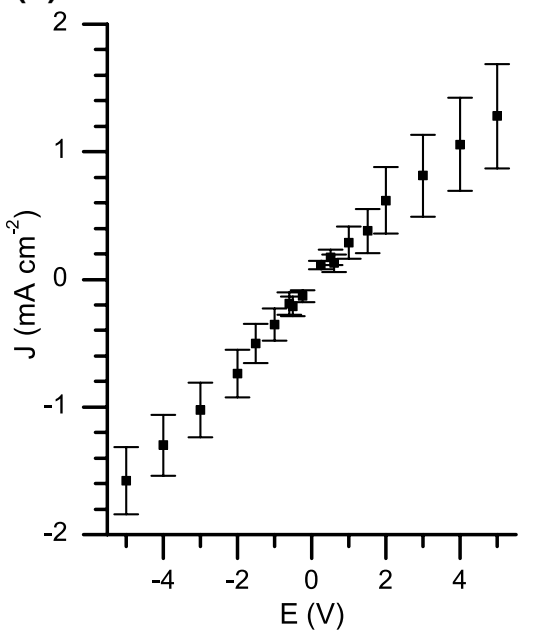


controlling potential (and measuring the current) or driving a specific current (and measuring the potential). However, controlling the potential makes it slightly more difficult to monitor the total charge transported compared to when driving constant current, making inadvertent operation beyond the coulombic capacity of the electrodes more likely. Any EOP, regardless of whether the electrodes are metal or conducting polymer, will eventually change the $\mathrm{pH}$ of the electrolyte if the transported charge exceeds the combined electrochemical capacity of the (electrochemically active) electrodes and the capacity of the $\mathrm{pH}$ buffer in the electrolyte. Tracking the transported charge allows the user to ensure that the EOP is operated within these limitations, minimizing undesired electrochemical reactions and their by-products. Operating an EOP at a constant current allows the total charge to be calculated as the simple product of the current (specified) and time, rather than requiring the measured current to be integrated over time as necessary when a constant voltage is used. Furthermore, a constant current, in a symmetric system, results in constant flow rate, even if accumulated charge at the electrodes or resistive losses in the system vary with time.

The limiting hydrostatic pressure $\Delta p_{\text {lim }}$ that the pump is capable of producing measured as a function of applied potential is shown in Fig. 6. We measured a maximum limiting hydrostatic pressure of $24.8 \pm 1.8 \mathrm{mmH}_{2} \mathrm{O} \mathrm{V}^{-1}$ or $0.25 \mathrm{kPa} \mathrm{V}^{-1}$, similar to what Wang et al. reported, even though their membranes had approximately double the pore diameter (Wang et al. 2012).

The potential supplied by the Keithley source measure unit is, of course, not the same as the difference in potential in the electrolyte on opposite sides of the membrane. This "transmembrane" potential was estimated by measuring

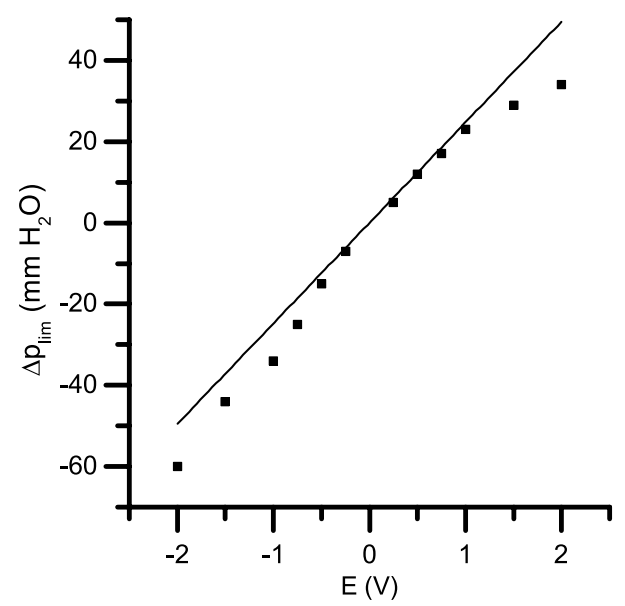

Fig. 6 Limiting hydrostatic pressure, $\Delta p_{\lim }$ versus applied potential $E$ measured for $1 \mathrm{mM} \mathrm{NaCl}$ with $\mathrm{pH} \sim 5.7 \pm 0.1$. The line represents the least squares fit through the origin, with a slope of $24.7 \pm 1.8$ $\mathrm{mmH}_{2} \mathrm{O} \mathrm{V} \mathrm{V}^{-1}$ the potential required to maintain zero current $(<100 \mathrm{pA})$ through two $\mathrm{Ag} / \mathrm{AgCl}$ reference electrodes, placed in each beaker, while operating the pump with a constant potential between the PEDOT electrodes. The transmembrane potential was measured to be 0.4 and $4.2 \mathrm{~V}$ with 1 and $5 \mathrm{~V}$, respectively, applied between the polymer electrodes, indicating that the resistive losses in the electrodes (including connections) and the combined potential drop across both electrode-electrolyte interfaces was between 0.6 and $0.8 \mathrm{~V}$.

Note that, during each period of operation, the potential "lost" at the electrodes increases as the electrochemical capacity of the PEDOT in each electrode is depleted. However, the polymer electrodes still far outperformed traditional metal electrodes. When we operated the pump with Pt electrodes instead of PEDOT electrodes, we did not generate measurable mass transport for applied potentials between 0.3 and $5 \mathrm{~V}$. Gas generation and $\mathrm{pH}$ changes were, however, detected. The Pt electrodes drove about one-tenth of the current observed when using PEDOT electrodes at the same applied potentials, further confirming that the reduction and oxidation of PEDOT is a much more effective electron-toion transducer for this EOP.

The electroosmotic mobility $\mu_{\mathrm{eo}}$ of the membrane was estimated using the results of the flow rate measurements using constant applied potentials (shown in Fig. 5a) as follows:

$\mu_{\mathrm{eo}}=Q L / \alpha A E$,

where $Q$ is the average volumetric flow rate, $L$ the membrane thickness, $A$ the area for flow through the membrane (estimated as the product of the pore cross-sectional area and number of pores), $E$ the effective applied potential (here assumed to be the full applied potential), and $\alpha$ is a correction factor that accounts for the decrease in effective pore radius due to the thickness of the double layer (Debye length). This equation is derived from the definition of electroosmotic mobility, assuming uniform and constant viscosity and electrical permittivity (Kirby 2010), and the value is proportional to the slope of the curve shown in Fig. 5a. This results in an uncorrected $(\alpha=1) \mu_{\mathrm{eo}}=(1.8 \pm 0.1) 10^{-}$ ${ }^{10} \mathrm{~m}^{2} \mathrm{~V}^{-1} \mathrm{~s}^{-1}$ for $1 \mathrm{mM} \mathrm{NaCl}$ at $\mathrm{pH}=5.7 \pm 0.1$. The Debye length for $1 \mathrm{mM} \mathrm{NaCl}$ is approximately $9.6 \mathrm{~nm}$, resulting in a correction factor $\alpha=0.9$ (Ai et al. 2010; Zeng et al. 2001), and a corrected $\mu_{\mathrm{eo}}=(2.0 \pm 0.1) 10^{-10} \mathrm{~m}^{2} \mathrm{~V}^{-1} \mathrm{~s}^{-1}$. The zeta potential $\zeta$, estimated from this electroosmotic mobility is as follows:

$\zeta=-\mu_{\mathrm{eo}} \eta /\left(\varepsilon_{r} \varepsilon_{0}\right)$,

where the solution viscosity $\eta$ is $1.01 \mathrm{cP}$ (Haynes et al. 2015) and the relative dielectric constant, $\varepsilon_{\mathrm{r}}$, is 80 , is $-0.29 \pm 0.01 \mathrm{mV}$ (Nörtemann et al. 1997). The results from the limiting hydrodynamic pressure measurement can also be used to calculate $\mu_{\mathrm{eo}}$, see (Zeng et al. 2001): 
$\Delta p_{\lim }=-8 \alpha \mu_{e o} \eta E / r^{2}$,

where $\Delta p_{\lim }$ is the limiting hydrodynamic pressure and $r$ the pore radius $(0.2 \mu \mathrm{m})$, resulting in $\zeta=-1.9 \pm 0.1 \mathrm{mV}$ (corrected with $\alpha=0.9$ ), which is much smaller in magnitude than reported by others, e.g., $-25 \mathrm{mV}$ at $\mathrm{pH}=6$, for both pure polycarbonate and PVP-treated polycarbonate membranes (Kirby and Hasselbrink 2004; Lettmann et al. 1999). Note that both $\zeta$ and $\mu_{\mathrm{eo}}$ should be calculated with the actual potential drop across the membrane, which we estimate to be about $75 \%$ of the actual applied potential, meaning that we have underestimated each by $\sim 33 \%$. The $\zeta$ we find appears to correspond to that of a polycarbonate/water interface with $\mathrm{pH}$ between 2.5 and 3.5. We cannot exclude the possibility that the inside of the pores has been contaminated by residues leached from the polymer electrodes. However, we do not observe a continuous decrease in pump performance over time of use of the membrane, indicating that contamination is unlikely to be the cause. The previously reported $\zeta$ indicates that this type of EOP should be able to generate even higher rates of flow and limiting pressures.

In general, the flow generated by the pump is proportional to the number of pores in the membrane, if all other parameters remain the same. As such, a more porous membrane would increase the pump's effect at a given applied potential. However, the method used to deposit the ions that form the pores does not disperse the ions evenly or uniformly (see Fig. 1). As such, increasing the pore density comes with the risk that adjacent pores will overlap, changing the effective pore diameter. Furthermore, the mechanical integrity of the membrane decreases with increasing pore density, which is crucial for textile applications where the membrane will be bent (flexed) repeatedly in comparison with traditional laboratory applications, where the membrane is fixed between rigid supports.

\section{Conclusions}

We have demonstrated a low-voltage, all-plastic, and flexible EOP made from a porous polycarbonate membrane and fabric electrodes coated with the electrochemically active polymer PEDOT. We report a maximum mass flux (based on total membrane cross-sectional area) of $23 \mathrm{mg} \mathrm{min}^{-1} \mathrm{~cm}^{-2}$ when operating the pump using potentials and currents similar to those that a small battery can supply. The use of the conductive polymer PEDOT together with a porous membrane results in a flexible structure through which water can be transported using potentials as low as $0.3 \mathrm{~V}$. Reduction and oxidation of PEDOT largely replaces water electrolysis as the electrochemical half-reactions, resulting in a nongassing and $\mathrm{pH}$-stable pump, even when used without a $\mathrm{pH}$ buffer. We hope that the combination of a non-mechanical, low-powered pump that is soft and flexible can find use in application areas where removal of water has previously been challenging, e.g., functional clothing and wound dressings.

Acknowledgements We thank Anders Elfwing for the SEM images. This work was funded by the Swedish Research Council (Vetenskapsrådet) Grant 2015-03298. NDR and KB have a stake in LunaMicro $\mathrm{AB}$, which owns a patent protecting the use of electrochemically active electrodes in electrokinetic devices.

Open Access This article is distributed under the terms of the Creative Commons Attribution 4.0 International License (http://creativecommons.org/licenses/by/4.0/), which permits unrestricted use, distribution, and reproduction in any medium, provided you give appropriate credit to the original author(s) and the source, provide a link to the Creative Commons license, and indicate if changes were made.

\section{References}

Ai Y, Yalcin SE, Gu DF, Baysal O, Baumgart H, Qian SZ, Beskok A (2010) A low-voltage nano-porous electroosmotic pump. J Colloid Interface Sci 350:465-470

Bard JA, Faulkner RL (2001) Electrochemical methods: fundamentals and applications. Wiley, New York

Bengtsson K, Nilsson S, Robinson ND (2014) Conducting polymer electrodes for gel electrophoresis. PLoS ONE. https://doi. org/10.1371/journal.pone.0089416

Brask A, Kutter JP, Bruus H (2005) Long-term stable electroosmotic pump with ion exchange membranes. Lab Chip 5:730-738. https://doi.org/10.1039/b503626g

Chen L, Ma J, Guan Y (2004) Study of an electroosmotic pump for liquid delivery and its application in capillary column liquid chromatography. J Chromatogr A 1028:219-226. https://doi. org/10.1016/j.chroma.2003.11.071

Eidsnes T, Ellingsen O, Heldal T, Mishchuk N (2004) Fabric material with improved liquid transport. PCT/NO2003/000247

Erlandsson PG, Robinson ND (2011) Electrolysis-reducing electrodes for electrokinetic devices. Electrophoresis 32:784-790. https:// doi.org/10.1002/elps.201000617

Glawdel T, Ren CL (2009) Electro-osmotic flow control for living cell analysis in microfluidic PDMS chips. Mech Res Commun 36:7581. https://doi.org/10.1016/j.mechrescom.2008.06.015

Haynes WM, Bruno TJ, Lide DR (2015) CRC handbook of chemistry and physics

Heldal T, Lauper D (2013) Liquid transport membrane. PCT/ EP2012/076527

Heuck FCA, Staufer U (2011) Low voltage electroosmotic pump for high density integration into microfabricated fluidic systems. Microfluid Nanofluid 10:1317-1332. https://doi.org/10.1007/ s10404-010-0765-2

Johansson T, Pettersson LAA, Inganäs O (2002) Conductivity of dedoped poly(3,4-ethylenedioxythiophene). Synth Metals 129:269274. https://doi.org/10.1016/S0379-6779(02)00086-3

Kirby BJ (2010) Micro- and nanoscale fluid dynamics, 1st edn. Cambridge University Press, New York

Kirby BJ, Hasselbrink EF (2004) Zeta potential of microfluidic substrates: 2. Data for polymers. Electrophoresis 25:203-213. https:// doi.org/10.1002/elps.200305755

Kumar R, Jahan K, Nagarale RK, Sharma A (2015) Nongassing Long-Lasting Electro-osmotic Pump with Polyaniline-wrapped 
Aminated Graphene Electrodes. ACS Appl Mater Interfaces 7:593-601. https://doi.org/10.1021/am506766e

Kwon K, Park C-W, Kim D (2012) High-flowrate, compact electroosmotic pumps with porous polymer track-etch membranes. Sensors Actuators A Phys 175:108-115. https://doi.org/10.1016/j. sna.2011.12.050

Lettmann C, Möckel D, Staude E (1999) Permeation and tangential flow streaming potential measurements for electrokinetic characterization of track-etched microfiltration membranes. J Membr Sci 159:243-251. https://doi.org/10.1016/S0376-7388(99)00067-8

Li PCH, Harrison DJ (1997) Transport, manipulation, and reaction of biological cells on-chip using electrokinetic effects. Anal Chem 69:1564-1568. https://doi.org/10.1021/ac9606564

Lin C-W, Yao S, Posner JD, Myers AM, Santiago JG (2007) Toward orientation-independent design for gas recombination in closedloop electroosmotic pumps. Sensors Actuators B Chem 128:334 339. https://doi.org/10.1016/j.snb.2007.05.029

Litster S, Suss ME, Santiago JG (2010) A two-liquid electroosmotic pump using low applied voltage and power. Sensor Actuators A Phys 163:311-314. https://doi.org/10.1016/j.sna.2010.07.008

Manz A, Effenhauser CS, Burggraf N, Harrison DJ, Seiler K, Fluri K (1994) Electroosmotic pumping and electrophoretic separations for miniaturized chemical analysis systems. J Micromech Microeng 4:257-265. https://doi.org/10.1088/0960-1317/4/4/010

Miao JY, Xu ZL, Zhang XY, Wang N, Yang ZY, Sheng P (2007) Micropumps based on the enhanced electroosmotic effect of aluminum oxide membranes. Adv Mater 19:4234

Nilsson D, Chen M, Kugler T, Remonen T, Armgarth M, Berggren $\mathrm{M}$ (2002) Bi-stable and dynamic current modulation in electrochemical organic transistors. Adv Mater 14:51-54. https:// dx.doi.org/10.1002/1521-4095(20020104)14:1\%3C51::AIDADMA51\%3E3.0.CO;2-\%23

Nörtemann K, Hilland J, Kaatze U (1997) Dielectric properties of aqueous $\mathrm{NaCl}$ solutions at microwave frequencies. J Phys Chem A. https://doi.org/10.1021/JP971623A

Sachan VK, Singh AK, Jahan K, Kumbar SG, Nagarale RK, Bhattacharya PK (2014) Development of redox-conducting polymer electrodes for non-gassing electro-osmotic pumps: a novel approach. J Electrochem Soc 161:H3029-H3034. https://doi. org/10.1149/2.0071413jes

Wang X, Cheng C, Wang S, Liu S (2009) Electroosmotic pumps and their applications in microfluidic systems. Microfluid Nanofluid 6:145-162. https://doi.org/10.1007/s10404-008-0399-9

Wang C, Wang L, Zhu X, Wang Y, Xue J (2012) Low-voltage electroosmotic pumps fabricated from track-etched polymer membranes. Lab Chip 12:1710-1716. https://doi.org/10.1039/c2lc40059f

Wu X, Ramiah Rajasekaran P, Martin CR (2016) An alternating current electroosmotic pump based on conical nanopore membranes. ACS Nano 10:4637-4643. https://doi.org/10.1021/acsnano.6b00939

Yao S, Hertzog DE, Zeng S, Mikkelsen JC, Santiago JG (2003) Porous glass electroosmotic pumps: design and experiments. J Colloid Interface Sci. https://doi.org/10.1016/S0021-9797(03)00730-6

Zeng SL, Chen CH, Mikkelsen JC, Santiago JG (2001) Fabrication and characterization of electroosmotic micropumps. Sensors Actuators B-Chem 79:107-114 